\title{
Inhibitor of DNA-binding 1 promotes endothelial progenitor cell proliferation and migration by suppressing E2-2 through the helix-loop-helix domain
}

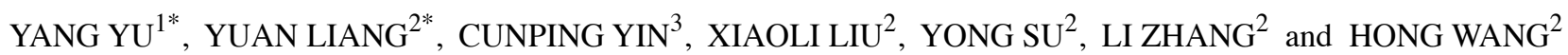 \\ ${ }^{1}$ Cardiology Center of PLA, Xinqiao Hospital, The Third Military Medical University, Chongqing, Sichuan 400037; \\ Departments of ${ }^{2}$ Geriatrics and ${ }^{3}$ Vascular Surgery, Kunming General Hospital of \\ Chengdu Military Command, Kunming, Yunnan 650032, P.R. China
}

Received October 2, 2015; Accepted August 10, 2016

DOI: $10.3892 /$ ijmm.2016.2734

\begin{abstract}
Vascular endothelial damage is the major contributing factor to cardiovascular diseases. Recently, the therapeutic significance of endothelial progenitor cells (EPCs) has drawn increasing attention due to their roles in re-endothelialization following injury. The inhibitor of DNA-binding 1 (ID1) has been proven to promote EPC proliferation and migration, suggesting a critical function of ID1 in re-endothelialization. However, the underlying mechanisms remain undefined. In this study, ID1 was found to interact with E2-2 using immunoprecipitation analysis. Moreover, ID1 overexpression suppressed E2-2 expression and luciferase reporter activity; however, these effects were not observed in cells transfected with ID1 lacking the helix-loop-helix (HLH) domain (ID1 $\Delta$ HLH). Further functional analysis corroborated that the upregulation of E2-2 markedly attenuated the ID1-mediated increase in EPC proliferation and migration. Furthermore, the HLH domain plays an important role in ID1-induced EPC proliferation and migration, as its deletion suppressed the positive regulatory effects of ID1 on EPC proliferation and migration. Taken together, the findings of our study confirm that ID1 promotes EPC proliferation and migration by suppressing E2-2 through the HLH domain in ID1. Therefore, ID1 may represent a potential therapeutic target for EPC-mediated re-endothelialization following vascular injury.
\end{abstract}

\section{Introduction}

Vascular endothelial injury is the pathological and physiological basis for cardiovascular diseases, including atherosclerosis and hypertension $(1,2)$. Recently, the enhancement of re-endotheli-

Correspondence to: Dr Hong Wang, Department of Geriatrics, Kunming General Hospital of Chengdu Military Command, 212 Daguan Road, Kunming, Yunnan 650032, P.R. China

E-mail: wanghongcnkm@163.com

Key words: inhibitor of DNA-binding 1, endothelial progenitor cell, cell proliferation, cell migration, helix-loop-helix domain alization has been recognized as a therapeutic option to repair injured vessels (3). During the process of re-endothelialization, it was previously considered that the adjacent endothelial cells (ECs) of injured vessels were the major contributors to the regeneration of the injured endothelium. However, their low proliferative potential limits their application in substituting the damaged endothelium following damage. Recently, emerging evidence has confirmed the important roles of endothelial progenitor cells (EPCs) in vascular injury due to their capacity to home to injury sites, and to differentiate into mature ECs to participate in re-endothelialization and angiogenesis following vascular injury (4-6). It has been reported that bone marrow (BM)-derived EPCs can control the angiogenic switch to induce tumor angiogenesis (7). Mounting evidence suggests that EPCs are the endogenous repair mechanism for maintaining the integrity of the endothelial monolayer (8). However, the underlying mechanisms involved in the regulation of EPC properties remain undefined.

Inhibitor of DNA-binding (ID) proteins are a helix-loophelix (HLH) family of transcription factors that play pivotal roles in various developmental processes, such as cell differentiation, development, migration and angiogenesis (9). As an important member of this family, ID1 has drawn increasing attention due to its important function in cell growth, migration and differentiation. ID1 lacks a DNA-binding domain, and can act as a dominant-negative regulator of the basic HLH (bHLH) transcription factors, which have been shown to be implicated in endothelial cell angiogenic activities (10). A previous study demonstrated the abundant expression of ID1 during blood vessel formation (11). ID1-knockout mice have been shown to exhibit abnormal angiogenesis (12). Moreover, the ectopic expression of ID1 has been shown to enhance EC proliferation and migration $(13,14)$. Recently, ID1 has been shown to positively regulate the proliferation and migration of spleen-derived EPCs (15). Furthermore, the silencing of ID1 reduces human ovarian cancer EPC angiogenesis (16). Although several studies have demonstrated the crucial role of ID1 in EPC angiogenesis, the mechanisms through which ID1 triggers angiogenesis remain poorly understood.

In this study, the ID1 interaction partners were analyzed using immunoprecipitation analysis, and the basic HLH 
transcription factor, E2-2, was identified. We investigated the mechanisms through which ID1 regulates E2-2 expression, as well as its role in ID1-indcued EPC proliferation and migration.

\section{Materials and methods}

Reagents and antibodies. Unless otherwise specified, all reagents used were from Sigma-Aldrich (St. Louis, MO, USA). The Dil-labeled acetylated low-density lipoprotein (Dil-Ac-LDL; \#BT-902) was obtained from Biomedical Technologies, Inc. (Stoughton, MA, USA). Fluorescein isothiocyanate-Ulex europaeus lectin-1 (FITC-UEA1; \#L9006) was obtained from Sigma-Aldrich. Fluorescein isothiocyanate (FITC)-conjugated antibodies against mouse stem cell antigen-1 (Sca-1; \#553335), vascular endothelial growth factor receptor 2 (VEGFR2; \#560680) and isotype control (\#553929) were purchased from BD Biosciences (San Diego, CA, USA). Rabbit polyclonal antibodies against mouse ID1 (ab52998) and E2-2 (ab185736) were obtained from Abcam (Cambridge, MA, USA). Antibodies against mouse Ki67 (sc-7846) and cyclin D1 (sc-753) were from Santa Cruz Biotechnology, Inc. (Santa Cruz, CA, USA). Rabbit monoclonals against p27 $7^{\mathrm{Kip} 1}$ (ab62364), $\beta$-actin (ab6276), matrix metalloproteinase (MMP)-2 (ab92536) and MMP-9 (ab58803) were from Abcam.

Preparation and identification of EPCs. Spleen-derived EPCs were isolated as previously described (15). Briefly, male Kunming mice ( $n=5$, weighing 25-30 g; Kunming General Hospital of Chengdu Military Command, Kunming, China) were employed to prepare the spleens. All mice were allowed to acclimatize for at least 2 weeks under normal husbandry conditions. The mice were then euthanized by an overdose of anesthesia with pentobarbital sodium (150 mg/kg, ip) and the tissues of the spleens were harvested aseptically by surgery. The animal experimental procedures were carried out after obtaining approval from the Institution Animal Care and Use Committee of Kunming General Hospital of Chengdu Military Command, Kunming, China. Total mononuclear cells from the spleens were isolated by density gradient centrifugation with Histopaque-1077 at $400 \mathrm{x}$ g for $20 \mathrm{~min}$. Approximately $24 \mathrm{~h}$ later, the unattached cells were discarded. The cells were then cultured in low-glucose Dulbecco's modified Eagle's medium (DMEM) supplemented with $10 \% \mathrm{FCS}$ and $10 \mathrm{ng} / \mathrm{ml}$ VEGF at $37^{\circ} \mathrm{C}$ with $5 \% \mathrm{CO}_{2}$. The medium was replaced every 2 days thereafter. After 4 days of culture, colony-forming cells were recognized as adherent cells, and were cultured continuously for further experiments. To identify the EPCs, the adherent cells were incubated with Dil-Ac-LDL $(10 \mathrm{mg} / \mathrm{ml})$ for $4 \mathrm{~h}$. Following fixation with $4 \%$ paraformaldehyde, the cells were then incubated with FITC-labeled lectin (UEA-1, $10 \mathrm{mg} / \mathrm{ml}$ ) for $1 \mathrm{~h}$. Dual-stained cells (positive for Dil-Ac-LDL and UEA-1) were identified as EPCs. Cellular nuclei were stained with DAPI (Sigma-Aldrich). The fluorescent images were recorded under a fluorescence microscope (IX51; Olympus, Tokyo, Japan).

Flow cytometric analysis. For further characterization of the EPCs, the dual-stained cells positive for Dil-Ac-LDL and UEA-1 were incubated with antibodies against mouse Sca-1 and VEGFR2 at $37^{\circ} \mathrm{C}$ with $5 \% \mathrm{CO}_{2}$. Approximately $30 \mathrm{~min}$ later, the cells were evaluated by flow cytometry using a
FACSAria flow cytometer (BD Immunocytometry Systems, Franklin Lakes, NJ, USA).

Adenoviruses and plasmids. Adenoviral vectors expressing Flag-IDl were prepared by inserting the coding sequence of ID1 into the pShuttle-CMV vector using the AdEasy system as previously described (17). After recombination of pShuttle-CMV-flag-ID1 with pADEasy-1, the obtained plasmids were transfected into 293T cells (ATCC, Manassas, VA, USA) to amplify the adenoviruses. EPCs were infected with recombinant ID1 adenovirus (AD-ID1) and empty vector (Ad). Cells without any treatment were defined as the control group. The adenoviralvector expressing Myc-E2-2 was also generated using the AdEasy system, as previously described (15).

The full-length cDNA of E2-2 was obtained by PCR, as previously described (18) and then cloned into the pMD19-T vector, followed by subcloning into pAdTrack-CMV to generate pAdTrack-E2-2. The resulting plasmids were transfected into 293T cells.

ID1 $\Delta$ HLH was generated by Pfx DNA polymerase (Invitrogen, Carlsbad, CA, USA) using mouse ID1 as a template with previously published methods (18).

Immunoprecipitation assay. To analyze the interaction between ID1 and E2-2, the prepared plasmids were transfected into COS7 cells (ATCC). Approximately $40 \mathrm{~h}$ later, the cells were lysed with lysis buffer. Following centrifugation, the supernatants were pre-cleared with protein G-Sepharose beads (GE Healthcare, Little Chalfont, Buckinghamshire, UK) for $30 \mathrm{~min}$ at $4^{\circ} \mathrm{C}$. The antibodies against Flag (Sigma-Aldrich) were then added for a further 2-h incubation. The protein complexes were immunoprecipitated by incubating with G-Sepharose Fast Flow beads, which had been pre-equilibrated in lysis buffer, for $30 \mathrm{~min}$. Beads collected by centrifugation were washed and resuspended in an equal volume of $5 \mathrm{X}$ sodium dodecyl sulfate (SDS) loading buffer. Immunoprecipitated proteins were separated by $10 \%$ SDS-PAGE, followed by transfer to Hybond-C Extra membranes. The membranes were incubated with anti-Myc 9E10 antibody (ab32; Abcam). For the detection of the endogenous interaction between ID1 and E2-2 in EPCs, cells were stimulated with bone morphogenetic protein 6 (BMP6; P20722; R\&D Systems; Minneapolis, MN, USA)) for $4 \mathrm{~h}$, and the immunoprecipitation was carried out with antibodies against E2-2 followed by western blot analysis with an anti-ID1 antibody.

RNA extraction and reverse transcription-quantitative polymerase chain reaction (RT-qPCR). The transfected EPCs were incubated for $48 \mathrm{~h}$ prior to RNA extraction. Total RNA was extracted using TRIzol reagent according to the manufacturer's instructions (Biostar, Shanghai, China). Subsequently, $5 \mu \mathrm{g}$ of total RNA of each sample was reverse transcribed into firststrand cDNA using the Promega Reverse Transcription System (Promega, Southampton, UK). The cDNA was used as the template for qPCR in a final volume of $20 \mu \mathrm{l}$ with the SYBR Premix Ex Taq II kit (Takara, Otsu, Japan). The following specific primers were used: ID1 sense, 5'-AGTGGTGCTTGG TCTGTCG-3' and antisense, 5'-GCAGGTCCCTGATGTAG TCG-3' and E2-2 sense, 5'-ATGGCTGCCTTAGGGACGG ACA-3' and antisense, 5'-AGGACCCTGAGCTACTTCTG-3'. $\beta$-actin was used as the endogenous control, and all results 
A

DAPI FITC-UEA1
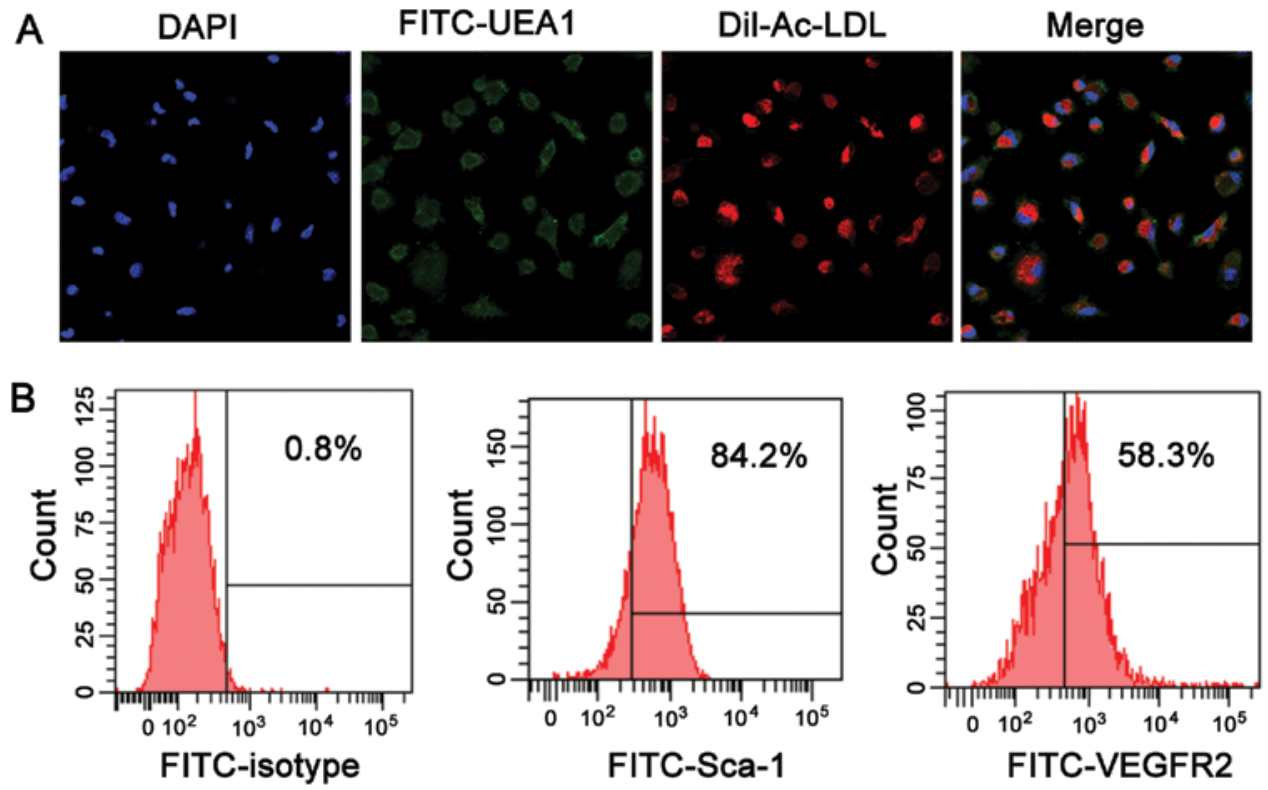

Figure 1. Phenotypic characterization of spleen-derived endothelial progenitor cells (EPCs). (A) Following 5-7 days in culture, the EPCs derived from the spleens of mice were stained with Dil-Ac-LDL and fluorescein isothiocyanate-conjugated Ulex europaeus lectin 1 (FITC-UEA1). The fluorescent images were recorded under a fluorescence microscope. (B) Flow cytometric analysis of cell markers of EPCs by detecting FITC-stem cell antigen-1 (Sca-1) and vascular endothelial growth factor receptor 2 (VEGFR2).

were calculated using the $2^{-\Delta \Delta C t}$ method, as previously described (19).

Western blot analysis. Following lysis with lysis buffer (Beyotime, Nantong, China), the protein concentrations were determined using the micro-BCA protein assay (Pierce, Rockford, IL, USA). The protein was separated by SDS-PAGE using a $12 \%$ polyacrylamide gel followed by electroblotting onto polyvinylidene difluoride (PVDF) membranes. After blocking the non-specific binding with 5\% non-fat milk, the membranes were incubated with the primary antibodies against ID1, E2-2, Ki67, p27 ${ }^{\text {Kip1 }}$, cyclin D1, MMP-9 and MMP-2. Horseradish peroxidase (HRP)-conjugated secondary antibodies were added for a further incubation for $1 \mathrm{~h}$. The binding signals were determined by electrogenerated chemilummescence (ECL) detection reagent (Beyotime). Blots against $\beta$-actin served as the loading control, and the results were normalized according to $\beta$-actin.

Transcriptional reporter assay. To examine the effects of ID1 on E2-2-induced transcription, the MCKpfos-luc reporter construct consisting of 4 E-box elements system was used as previously described (20). Briefly, the EPCs were seeded at a density of $5 \times 10^{4}$ cells/well in 24 -well plates 1 day prior to transfection. The cells were then transfected with MCKpfos-luc, E2-2, and either Ad-ID1 or ID1 $\triangle H L H$ using Lipofectamine (Life Technologies, Carlsbad, CA, USA). Approximately $40 \mathrm{~h}$ later, the lysates were collected and were subsequently analyzed for luciferase activity using a luciferase assay system (Promega, Madison, WI, USA).

3-(4,5-Dimethylthiazol-2-yl)-2,5-diphenyltetrazolium bromide (MTT) assay. The EPCs were collected and seeded into 96-well plates at a density of $2 \times 10^{6}$ cells/well. After preconditioning with the indicated treatments, the cells were cultured with fresh medium containing $15 \mu \mathrm{MTT}$ reagent for a further $5 \mathrm{~h}$ at $37^{\circ} \mathrm{C}$.
The supernatant was then discarded, and $200 \mu 1$ of DMSO were added to each well to dissolve the formazan. The absorbance at $490 \mathrm{~nm}$ was measured to analyze cell proliferation using a micro-enzyme-linked immunosorbent assay (ELISA) reader (Bio-Rad, Hercules, CA, USA).

Cell migration assay. The migration of EPCs was analyzed by a modified Boyden's chamber assay as previously described (15). Briefly, the cells (1x10\%/well in $200 \mu 1$ serum-free medium) were seeded into the upper chamber. DMEM medium containing $10 \% \mathrm{FCS}$ and $50 \mathrm{ng} / \mathrm{ml}$ VEGF was added to the lower chamber as the chemoattractant. Approximately $8 \mathrm{~h}$ later, the non-migrating cells on the upper surface of the $8-\mu \mathrm{m}$ filters were removed with a cotton swab. Cells that had penetrated to the lower surface were fixed with $4 \%$ paraformaldehyde and stained with $0.1 \%$ crystal violet. The cell migration ability was evaluated by counting cells in 5 randomly selected visual fields with an inverted microscope at x100 magnification.

Statistical analysis. All results are presented as the means \pm SD of at least 3 independent measurements in triplicate. SPSS 13.0 software was used to analyze all data. Statistically significant differences between different groups were determined based on the Student's t-test and ANOVA (analysis of variance). A value of $\mathrm{P}<0.05$ was considered to indicate a statistically significant difference.

\section{Results}

Characterization of spleen-derived EPCs. After 5-7 days of culture, the attached EPCs derived from the spleens of mice exhibited a spindle-shaped morphology. EPCs are characterized by being double positive for Dil-Ac-LDL uptake and lectin binding (Fig. 1A). FACS analysis revealed expression of the stem cell marker, Sca-1, and the endothelial marker, VEGFR2. 


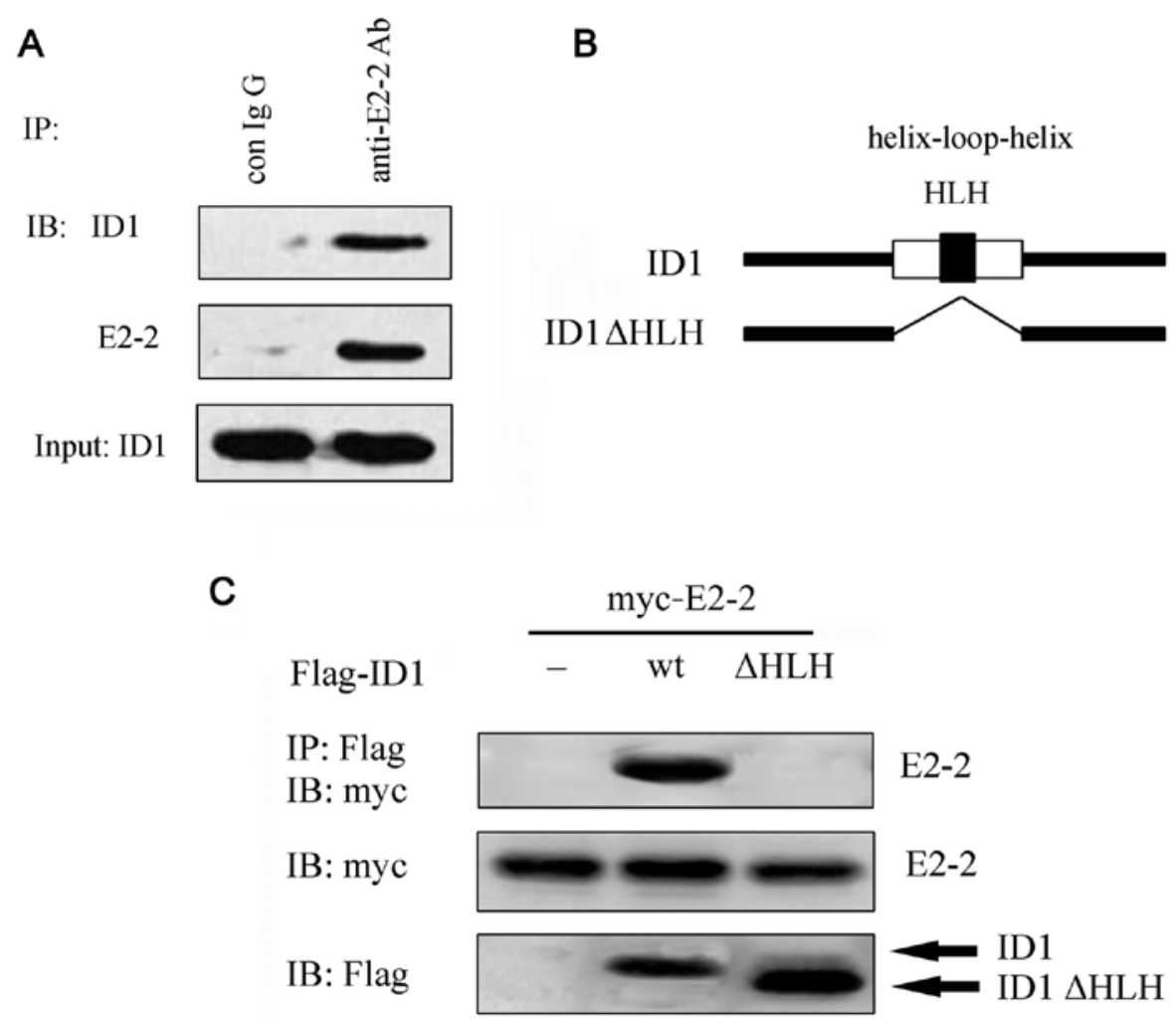

Figure 2. Interaction of E2-2 and inhibitor of DNA-binding 1 (ID1). (A) Following stimulation with bone morphogenetic protein 6 (BMP6) for 4 h, cell lysates from endothelial progenitor cells (EPCs) were immunoprecipitated with anti-E2-2 antibody or control IgG. Western blot analysis was performed to detect the endogenous interaction between ID1 and E2-2 with antibodies against E2-2 and ID1. (B) The schematic diagrams of helix-loop-helix (HLH)-containing widetype (WT) and deletion mutants of ID1. (C) Interaction of Myc-E2-2 and Flag-ID1 via the HLH domain. Myc-E2-2-transfected Cos7 cells were co-transfected with Flag-ID1 (wt) or ID1 $\Delta$ HLH. Expression of Flag-ID1 and Myc-E2-2 proteins was analyzed by anti-Flag M5 antibodies and anti-Myc 9E10 antibodies, respectively.

The Sca-1-positive cells accounted for $84.2 \pm 3.6 \%$ of the cells, while the VEGFR2-positive cells accounted for $58.3 \pm 4.1 \%$ of the cells (Fig. 1B).

Identification of E2-2 as an interaction partner of IDI. To determine the mechanisms through which ID1 regulates EPC proliferation and migration, we investigated the protein of E2-2, which has been proven to interact with human ID1 using a yeast two-hybrid system in a previous study (18). To demonstrate the interaction between endogenous ID1 and E2-2, the EPCs were stimulated with BMP6 for $4 \mathrm{~h}$ to induce the expression of ID1. Following lysis and immunoprecipitation, western blot analysis revealed that endogenous ID1 formed a complex with endogenous E2-2 in the EPCs (Fig. 2A). Moreover, following transfection with Myc-E2-2 and Flag-ID1 into the COS7 cells, the complex between ID1 and E2-2 was also observed (Fig. 2C). It is known that ID HLH proteins often act as dominant-negative regulators of bHLH transcriptional regulators. To further elucidate the mechanisms through which ID1 interacts with E2-2, we constructed ID1 mutants lacking the HLH domains (Fig. 2B). An immunoprecipitation assay revealed that E2-2 did not interact with ID1 $\Delta$ HLH. Taken together, these results suggest that ID1 heterodimerizes with E2-2 via its HLH domain.

ID1 suppresses the expression of E2-2 and E2-2-mediated transcription. To examine the effects of the ID1-E2-2 interaction on the E2-2 levels, an adenoviral vector carrying ID1 (Ad-ID1) was constructed. Following the transfection of
Ad-ID1 into EPCs, the mRNA levels of ID1 were upregulated $(\mathrm{P}<0.05 ;$ Fig. 3A), accompanied by a similar upregulation in its protein levels $(\mathrm{P}<0.05$; Fig. 3B). The results of RT-qPCR revealed that the overexpression of ID1 markedly suppressed the mRNA levels of E2-2 ( $\mathrm{P}<0.05$; Fig. 3C), as well as its protein levels ( $\mathrm{P}<0.05$; Fig. 3D). To further examine the effects of ID1 on E2-2, the MCKpfos-luc reporter construct containing 4 E-box elements was transfected into the EPCs. As one of the E-proteins, E2-2 transfection enhanced the reporter activity $(\mathrm{P}<0.05 ;$ Fig. $3 \mathrm{E})$. However, the upregulation of ID1 significantly blocked E2-2induced MCKpfos-luc reporter activity $(\mathrm{P}<0.05 ; \mathrm{Fig}$. 3E). The above-mentioned data verified that ID1 inhibited the expression and transcriptional activity of E2-2.

HLH-dependent inhibition of E2-2 by IDI. In order to investigate the mechanisms responsible for the regulation of E2-2 by ID1, the functional role of its HLH motif was analyzed. As shown in Fig. 2B, the deletion mutants of ID1 were constructed and transfected into the EPCs. The results of RT-qPCR revealed that the upregulation of ID1 inhibited the mRNA levels of E2-2 $(\mathrm{P}<0.05)$; however, this did not occur in the cells transfected with ID1 $\triangle \mathrm{HLH}$ (Fig. 3C). Similarly, the corresponding decrease in the E2-2 protein levels triggered by the overexpression of ID1 was attenuated when the cells were transfected with ID1 $\triangle H L H ~(P<0.05)$. The deletion of the HLH domain in ID1 did not suppress the MCKpfos-luc reporter activity induced by E2-2 (Fig. 3E), suggesting that ID1 inhibited E2-2 expression and transcriptional regulation via the HLH motif. 
A

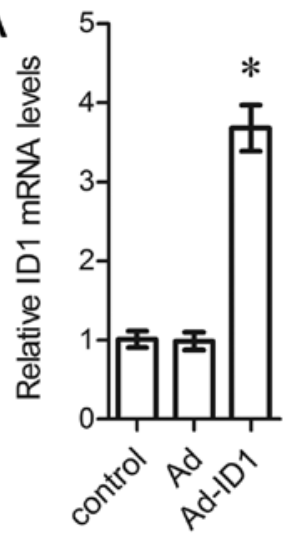

C

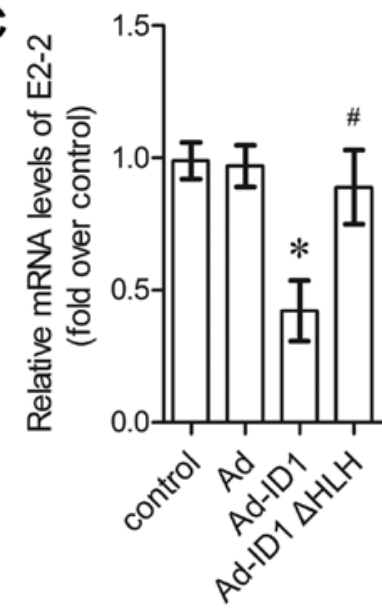

$E$

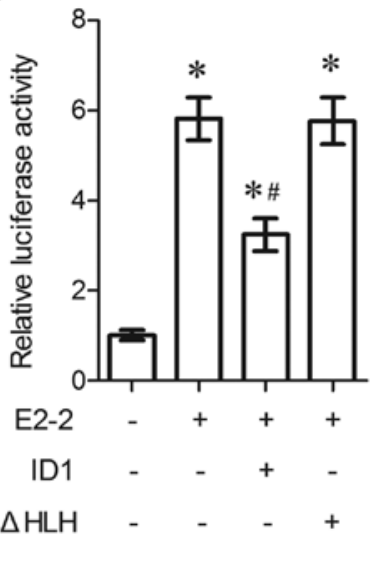

B
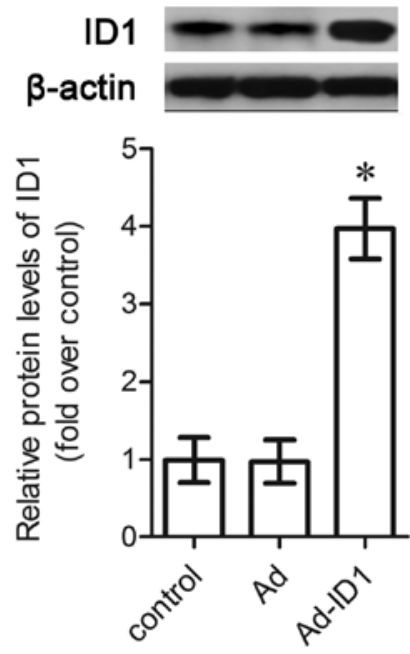

D E2-2

$\beta$-actin

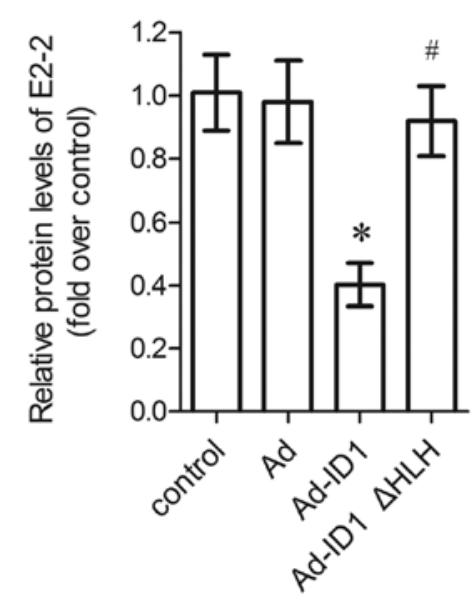

Figure 3. Inhibitor of DNA-binding 1 (ID1) inhibits E2-2 expression and E2-2-mediated luciferase activity. (A) Endothelial progenitor cells (EPCs) were infected with Ad-ID1, and the mRNA levels of ID1 were analyzed by RT-qPCR. * $\mathrm{P}<0.05$ vs. control groups. (B) The effect of Ad-ID1 infection on ID1 protein expression was evaluated by western blot analysis. ${ }^{*} \mathrm{P}<0.05$. (C) Following infection with Ad-ID1, the effect of ID1 overexpression on E2-2 mRNA levels was determined by RT-qPCR. (D) Effect of ID1 upregulation on E2-2 protein expression. ${ }^{*} \mathrm{P}<0.05$ vs. control groups; ${ }^{\text {P }}<0.05$ vs. Ad-ID1-transfected groups. (E) EPCs were transfected with MCKpfos-luc, E2-2, and ID1 or ID1 $\Delta$ HLH. Lysates were collected and luciferase activity was assessed using a luciferase assay system. ${ }^{*} \mathrm{P}<0.05$ vs. control groups; ${ }^{\#} \mathrm{P}<0.05$ vs. E2-2-transfected only groups.

E2-2 is responsible for ID1-induced EPC proliferation. EPC-based gene therapy can contribute to re-endothelialization and can inhibit intimal hyperplasia following vascular injury (5). Our previous study demonstrated the important role of ID1 in regulating EPC proliferation and migration (15). In this study, to investigate the underlying mechanisms, we examined the effect of E2-2 during this processs. Following infection with Ad-E2-2, the expression levels of E2-2 were significantly upregulated $(\mathrm{P}<0.05$; Fig. 4A). The upregulation of E2-2 antagonized EPC proliferation and induced a time-dependent inhibition of cell proliferation (Fig. 4B). To further clarify the role of E2-2 in ID1-induced cell proliferation, MTT assay was carried out and the results corroborated that ID1 enhanced EPC proliferation, which was attenuated by E2-2 upregulation ( $\mathrm{P}<0.05$; Fig. 4C). Simultaneously, the expression of the cell proliferation marker, Ki67, indcued by ID1 was downregulated in the cells transfected with Ad-E2-2 ( $\mathrm{P}<0.05$; Fig. 4D and E). Moreover, the inhibitory effects of ID1 on the expression of proliferation-related cyclindependent kinase $(\mathrm{Cdk})$ inhibitor $\mathrm{p} 27^{\mathrm{Kip} 1}$ were also abated in the Ad-E2-2-transfected cells, concomitant with a decrease in cyclin D1 expression ( $\mathrm{P}<0.05$; Fig. 4D and E).

IDI induces EPC migration by suppressing E2-2. As shown in Fig. 5A, the upregulation of E2-2 suppressed EPC migration $(\mathrm{P}<0.05)$, and the overespression of ID1 significantly increased the number of migrated EPC cells ( $\mathrm{P}<0.05$; Fig. 5B). However, this increase in cell migration was significantly attenuated by the overexpression of E2-2 (P<0.05; Fig. 5B).

Both MMP-2 and MMP-9 are associated with cell migration $(16,21)$. The results of western blot analysis revealed that the enhanced expression of MMP-2 and MMP-9 induced by ID1 were also decreased in the E2-2-overexpressing cells $(\mathrm{P}<0.05$; Fig. 5C and D). Overall, the above-mentioned results confirm that ID1 enhances EPC migration in an E2-2dependent manner.

The HLH domain is critical for IDI-induced EPC proliferation and migration. Our above-mentioned findings demonstrated 
A

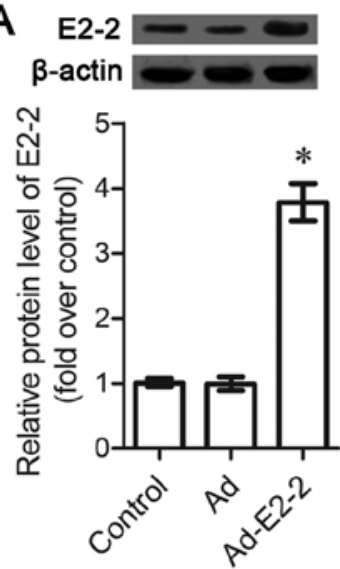

B

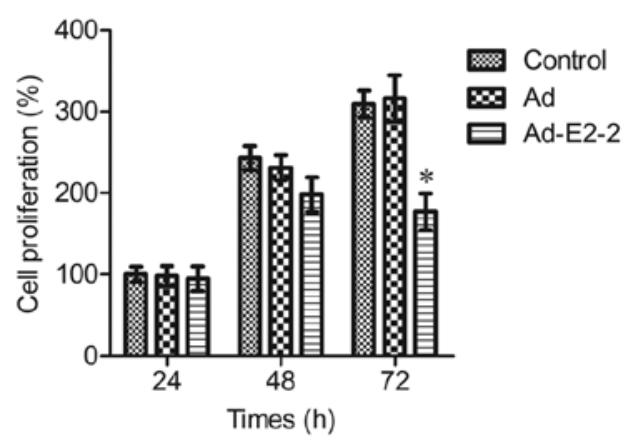

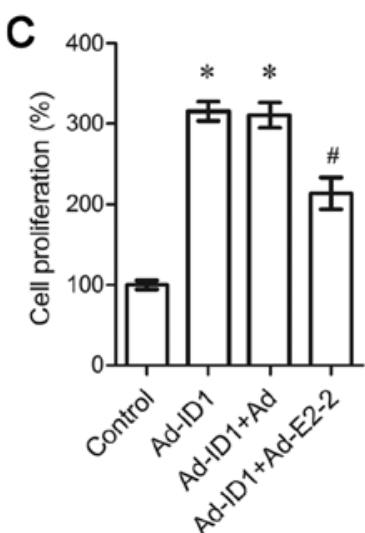
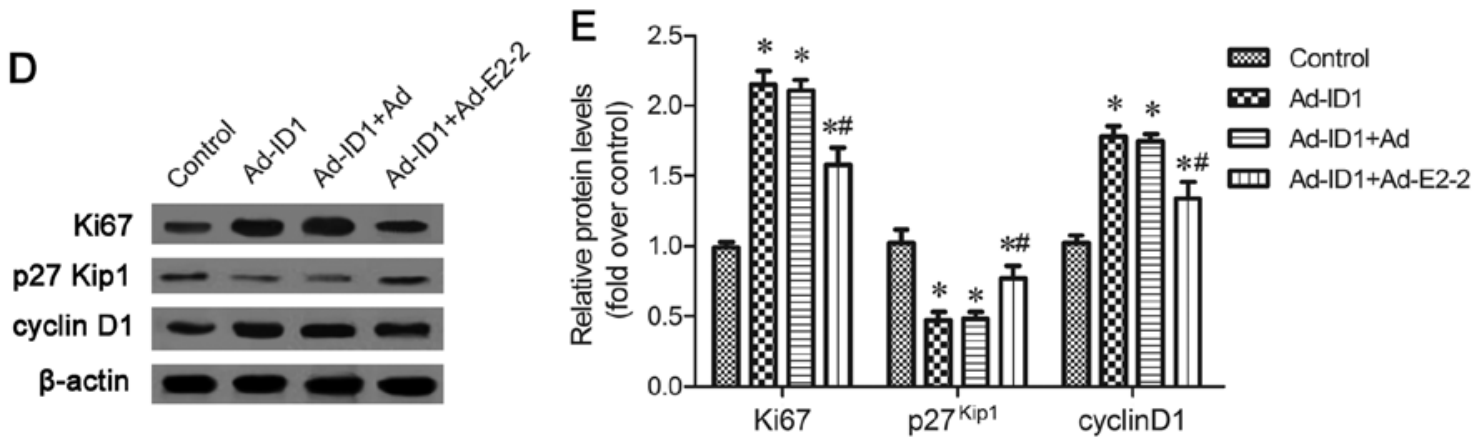

Figure 4. Inhibitor of DNA-binding 1 (ID1)-induced cell proliferation is attenuated by E2-2 overexpression. (A) Endothelial progenitor cells (EPCs) were infected with Ad-E2-2. The effect of Ad-E2-2 infection on E2-2 protein levels was detected by western blot analysis. (B) Following transfection for the indicated periods of time, cell proliferation was examined by MTT assay by detecting the absorbance at $490 \mathrm{~nm}$. (C) Ad-ID1 pre-infection cells were infected with Ad-E2-2. The effect of Ad-E2-2 infection on ID1-triggered cell proliferation was evaluated by MTT assay. (D) E2-2 upregulation relieves the effect of ID1 on the expression of cell proliferation-related molecules. Cells were transfected with Ad-ID1 followed by E2-2 transfection. The protein levels of Ki67, p27 ${ }^{\text {Kipl }}$ and cyclin D1 were detected by western blot analysis. (E) Quantitative analysis of Ki67, p27 ${ }^{\mathrm{Kipl}}$ and cyclin D1 protein levels. " $\mathrm{P}<0.05$ vs. control groups; ${ }^{*} \mathrm{P}<0.05$ vs. Ad-ID1transfected groups.

the important role of the HLH motif in E2-2 expression. However, its role in ID1 function remains unclear. To further clarify the function of the HLH domain, we constructed ID1 mutants lacking the respective HLH domains. The reslts of MTT assay revealed that ID1 overexpression induced EPC proliferation; however, this increase was significantly inhibited in the ID1 $\Delta$ HLH-transfected cells ( $\mathrm{P}<0.05$; Fig. 6A). Moreover, similar changes in proliferation-related proteins were also observed, including Ki67 and cyclin D1. The expression of $\mathrm{p} 27^{\mathrm{Kip} 1}$ was inhibited by the overexpression of ID1 and it was increased in the ID1 $\Delta$ HLH-transfected cells $(\mathrm{P}<0.05$; Fig. 6C and D). The enhanced number of cells that had migrated due to ID1 upregulation was also blocked when ID1 lacked the HLH motif ( $\mathrm{P}<0.05$; Fig. 6B), which was accompanied by corresponding changes in the expression of MMP-2 and MMP-9 ( $\mathrm{P}<0.05$; Fig. 6C and D). On the whole, these results suggest that the HLH domain is pivotal in ID1-induced EPC proliferation and migration.

\section{Discussion}

Endothelial damage is a major contributor to atherosclerosis and other cardiovascular diseases (22). Recently, the therapeutic significance of EPCs has drawn increasing attention due to their critical function in re-endothelialization $(4,5)$. EPCs are recognized as the major source of cells participating in endothelial repair and subsequently, re-endothelialization following vascular injury. Their number and function are inversely correlated with the risk factors for coronary artery disease $(23,24)$. Increasing evidence suggests that the proliferation and migration of EPCs is the key mechanism in re-endothelialization following vascular injury (25-28). Therefore, understanding the mechanisms involved in re-endothelialization by EPCs will lead to novel strategies for the treatment of vascular endothelial injury-related diseases.

ID1 is a critical subfamily member of the HLH proteins, and plays a pivotal role in angiogenesis (11). The gigh expression of ID1 has been observed during vascular formation (11), and it can enhance EC proliferation and migration (14). Compared with healthy patients, ID1 expression is increased in ovarian cancer patients, and its silencing substantially reduces EPC angiogenesis (16). Our previous study demonstrated that ID1 overexpression promoted EPC proliferation and migration, indicating a vital role for ID1 in re-endothelialization (15). However, its underlying mechanisms of action remain unclear. In this study, we searched for ID1 interaction partners using immunoprecipitation analysis and found that ID1 formed a complex with E2-2. Moreover, ID1 upregulation inhibited the expression of E2-2 and the E2-2-induced activity of an artificial E-box-containing reporter (MCKpfos-luc). Therefore, these results suggest that ID1 interacts with E2-2. However, 

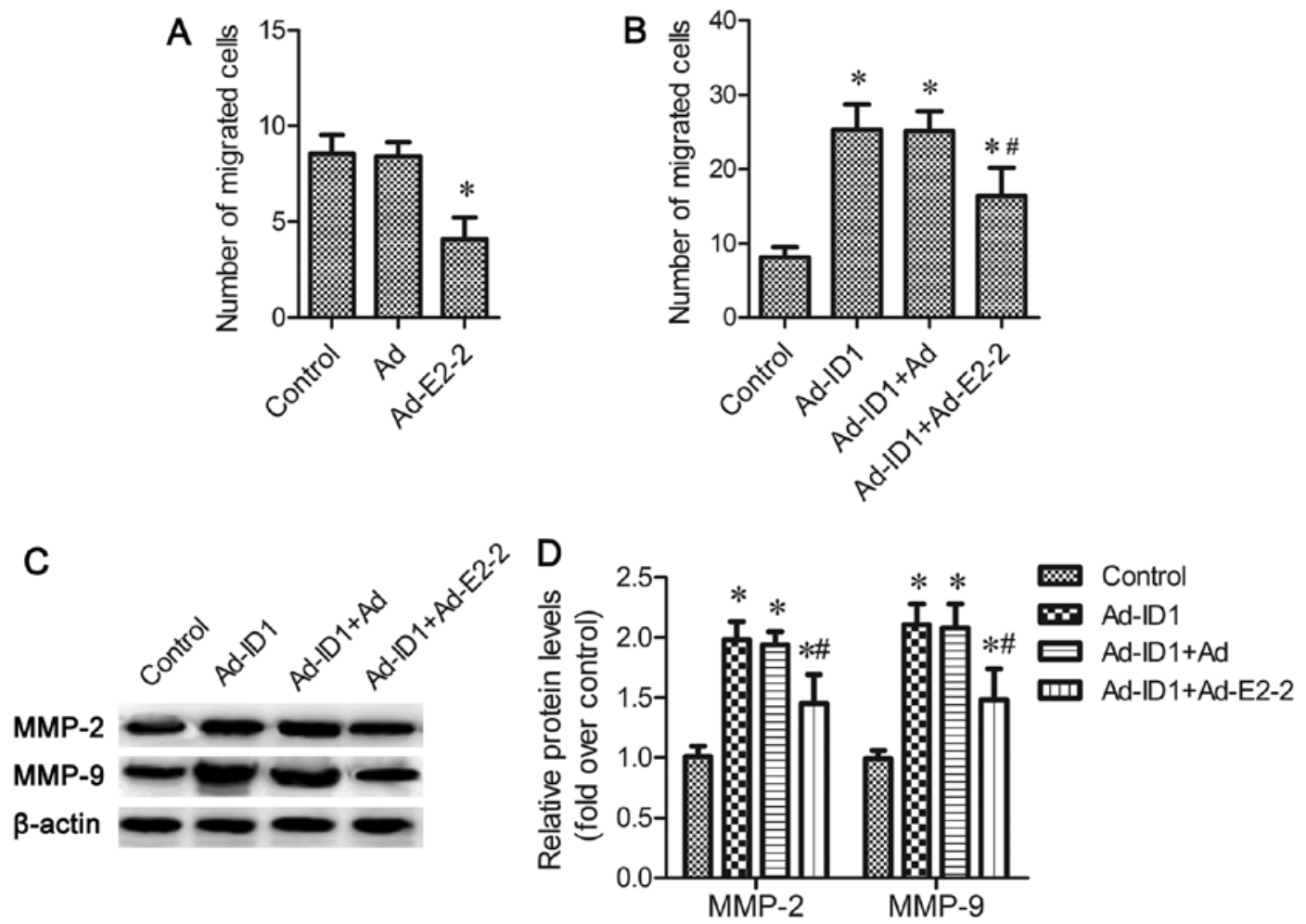

Figure 5. Inhibitor of DNA-binding 1 (ID1)-induced cell migration is attenuated by E2-2 overexpression. (A) endothelial progenitor cells (EPCs) were infected with Ad-E2-2. Cell migration was observed using a modified Boyden's chamber assay. (B) EPCs pre-infected with AD-ID1 were infected with Ad-E2-2. Cell migration was analyzed. (C) The role of E2-2 in the ID1-induced overexpression of MMP-2 and MMP-9 was determined by western blot analysis. (D) Quantitative assay of MMP-2 and MMP-9 proteins. ${ }^{*} \mathrm{P}<0.05$ vs. control groups; ${ }^{*} \mathrm{P}<0.05$ vs. Ad-ID1-transfected groups.
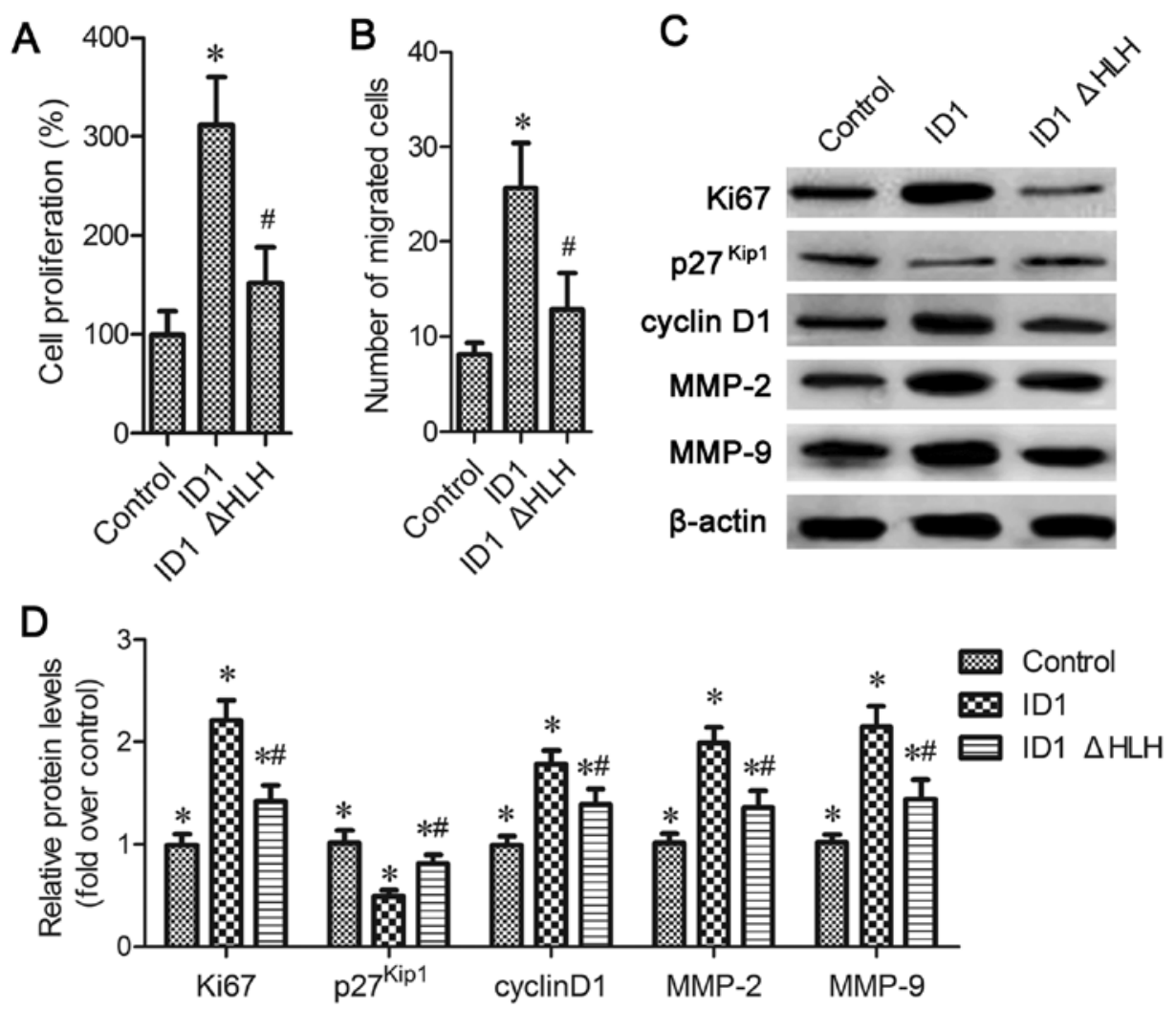

Figure 6. The helix-loop-helix (HLH) domain is critical for inhibitor of DNA-binding 1 (ID1)-induced endothelial progenitor cell (EPC) proliferation and migration. To investigate the function of the HLH motif on ID1-triggered cell proliferation and migration, cells were infected with Ad-ID1 or ID1 mutants lacking the respective HLH domains. (A) Cell proliferation was monitored by MTT assay. (B) Cell migration was analyzed using a modified Boyden's chamber assay. (C) The effect of HLH domain on the expression of Ki67, p27 $7^{\mathrm{Kipl}}$ and cyclin D1 was examined by western blot analysis. (D) Quantitative analysis of Ki67, p27 ${ }^{\mathrm{Kipl}}$ and cyclin $\mathrm{D} 1$ protein levels. ${ }^{*} \mathrm{P}<0.05$ vs. control groups; ${ }^{*} \mathrm{P}<0.05$ vs. Ad-ID1-transfected groups. 
whether E2-2 plays an important role in ID1-induced EPC function needs to be explored further.

E2-2, which is also known as TCF4, belongs to the E-protein family or the class-A type of bHLH transcription factors that are involved in various physiological processes, such as cellular growth, differentiation, and neural development (29-31). Previous studies have demonstrated that ID proteins lack the basic DNA-binding domain, and can act as a dominant-negative regulator of bHLH to regulate cell commitment, differentiation and embryogenesis by forming inactive hererodimers $(9,10)$. Recently, E2-2 was shown to repress VEGFR2 reporter activity, endothelial cell activation and subsequent angiogenesis $(10,20)$. In this study, we demonstrated the inhibitory effect of ID1 on E2-2 levels. To further clarify the underlying mechanisms involved in ID1-regulated EPC proliferation and migration, we investigated the function of E2-2. E2-2 overexpression inhibited EPC proliferation, accompanied by a corresponding decrease in the expression of the cell proliferation marker, Ki67, and cell cycle-related proteins (cyclin D1), and an increase in p27 expression. Moreover, E2-2 upregulation also attenuated cell migration, concomitant with a downregulation in MMP-2 and MMP-9 levels. Importantly, the enhanced effects of ID1 on EPC proliferation and migration were attenuated when the cells were transfected with Ad-E2-2. These data indicate that ID1 may trigger EPC angiogenesis by enhancing cell proliferation and migration through E2-2.

ID proteins often bind to E-proteins through the HLH motif to inhibit the corresponding transcription (9). In this study, we demonstrated the critical role of the HLH domain in the interaction between ID1 and E2-2. Furthermore, the lack of HLH exhibited little effect on E2-2-induced MCKpfos-luc transcriptional activity. Importantly, the inhibitory effect of ID1 on E2-2 expression was abolished when the cells were transfected with ID1 that lacked the HLH domain. It has been demonstrated that blocking ID1 function by dominant interfering HLH dimerization mutant 13I represses angiogenic factor vascular endothelial growth factor (32). To further investigate the underlying mechanisms involved in ID1-induced EPC proliferation and migration by E2-2, we analyzed the function of HLH during these processes. As expected, the enhanced effect of ID1 on EPC proliferation was notably attenuated when the HLH domain in ID1 was deleted. A similar effect on cell migration was also observed. These results allow us to speculate that the HLH domain is critical for ID1-induced EPC proliferation and migration via E2-2.

In conclusion, this study demonstrates that ID1 interacts with E2-2 in EPCs. Importantly, ID1 exerted its positive regulatory effect on EPC proliferation and migration via E2-2 and its HLH domain. Therefore, this study indicates a potential role for ID1 in the development of the re-endothelialization processes based on EPCs, and supports a promising therapeutic option for repairing injured blood vessels.

\section{Acknowledgements}

This study was supported by the National Natural Science Foundation of China (no. 81270224) and the Chengdu military region's 12th five foundation (no. C12053).

\section{References}

1. Sena CM, Pereira AM and Seiça R: Endothelial dysfunction - a major mediator of diabetic vascular disease. Biochim Biophys Acta 1832: 2216-2231, 2013.

2. Hirase T and Node K: Endothelial dysfunction as a cellular mechanism for vascular failure. Am J Physiol Heart Circ Physiol 302: H499-H505, 2012.

3. $\mathrm{Hu} \mathrm{CH}, \mathrm{Ke} \mathrm{X}$, Chen $\mathrm{K}$, Yang DY, Du ZM and Wu GF: Transplantation of human umbilical cord-derived endothelial progenitor cells promotes re-endothelialization of the injured carotid artery after balloon injury in New Zealand white rabbits. Chin Med J (Engl) 126: 1480-1485, 2013.

4. Balaji S, King A, Crombleholme TM and Keswani SG: The role of endothelial progenitor cells in postnatal vasculogenesis: Implications for therapeutic neovascularization and wound healing. Adv Wound Care (New Rochelle) 2: 283-295, 2013.

5. Zhang M, Malik AB and Rehman J: Endothelial progenitor cells and vascular repair. Curr Opin Hematol 21: 224-228, 2014.

6. Yu J, Wang Q, Wang H, Lu W, Li W, Qin Z and Huang L: Activation of liver $X$ receptor enhances the proliferation and migration of endothelial progenitor cells and promotes vascular repair through PI3K/Akt/eNOS signaling pathway activation. Vascul Pharmacol 62: 150-161, 2014.

7. Dudley AC, Cloer EW and Melero-Martin JM: The role of bone marrow-derived progenitor cells in tumor growth and angiogenesis. In: Stem Cells and Cancer Stem Cells. Vol. 8. Springer, pp45-54, 2012.

8. Hibbert B, Ma X, Pourdjabbar A, Holm E, Rayner K, Chen YX, Sun J, Filion L and O'Brien ER: Inhibition of endothelial progenitor cell glycogen synthase kinase- $3 \beta$ results in attenuated neointima formation and enhanced re-endothelialization after arterial injury. Cardiovasc Res 83: 16-23, 2009.

9. Norton JD: ID helix-loop-helix proteins in cell growth, differentiation and tumorigenesis. J Cell Sci 113: 3897-3905, 2000.

10. Tanaka A, Itoh F, Itoh S and Kato M: TAL1/SCL relieves the E2-2-mediated repression of VEGFR2 promoter activity. J Biochem 145: 129-135, 2009.

11. Benezra R, Rafii S and Lyden D: The Id proteins and angiogenesis. Oncogene 20: 8334-8341, 2001

12. Lyden D, Young AZ, Zagzag D, Yan W, Gerald W, O'Reilly R, Bader BL, Hynes RO, Zhuang Y, Manova K and Benezra R: Id1 and Id 3 are required for neurogenesis, angiogenesis and vascularization of tumour xenografts. Nature 401: 670-677, 1999.

13. Valdimarsdottir G, Goumans M-J, Rosendahl A, Brugman M, Itoh S, Lebrin F, Sideras P and ten Dijke P: Stimulation of Id1 expression by bone morphogenetic protein is sufficient and necessary for bone morphogenetic protein-induced activation of endothelial cells. Circulation 106: 2263-2270, 2002.

14. Ling M-T, Lau TC, Zhou C, Chua CW, Kwok WK, Wang Q, Wang $X$ and Wong YC: Overexpression of Id-1 in prostate cancer cells promotes angiogenesis through the activation of vascular endothelial growth factor (VEGF). Carcinogenesis 26: 1668-1676, 2005

15. Wang H, Yu Y, Guo RW, Shi YK, Song MB, Chen JF, Yu SY, Yin YG, Gao $P$ and Huang L: Inhibitor of DNA binding-1 promotes the migration and proliferation of endothelial progenitor cells in vitro. Mol Cell Biochem 335: 19-27, 2010.

16. Su Y, Gao L, Teng L, Wang Y, Cui J, Peng S and Fu S: Id1 enhances human ovarian cancer endothelial progenitor cell angiogenesis via PI3K/Akt and NF-кB/MMP-2 signaling pathways. J Transl Med 11: 132, 2013

17. Itoh F, Itoh S, Goumans MJ, Valdimarsdottir G, Iso T, Dotto GP, Hamamori Y, Kedes L, Kato M and ten Dijke Pt P: Synergy and antagonism between Notch and BMP receptor signaling pathways in endothelial cells. EMBO J 23: 541-551, 2004.

18. Tanaka A, Itoh F, Nishiyama K, Takezawa T, Kurihara H, Itoh S and Kato M: Inhibition of endothelial cell activation by bHLH protein E2-2 and its impairment of angiogenesis. Blood 115: 4138-4147, 2010.

19. Livak KJ and Schmittgen TD: Analysis of relative gene expression data using real-time quantitative PCR and the 2(-Delta Delta C(T)) Method. Methods 25: 402-408, 2001.

20. Yang W, Itoh F, Ohya H, Kishimoto F, Tanaka A, Nakano N, Itoh S and Kato M: Interference of E2-2-mediated effect in endothelial cells by FAM96B through its limited expression of E2-2. Cancer Sci 102: 1808-1814, 2011.

21. Von Offenberg Sweeney N, Cummins PM, Cotter EJ, Fitzpatrick PA, Birney YA, Redmond EM and Cahill PA: Cyclic strain-mediated regulation of vascular endothelial cell migration and tube formation. Biochem Biophys Res Commun 329: $573-582,2005$. 
22. Cai $\mathrm{H}$ and Harrison DG: Endothelial dysfunction in cardiovascular diseases: The role of oxidant stress. Circ Res 87: 840-844, 2000.

23. Pearson JD: Endothelial progenitor cells - hype or hope? J Thromb Haemost 7: 255-262, 2009.

24. Vasa M, Fichtlscherer S, Aicher A, Adler K, Urbich C, Martin H, Zeiher AM and Dimmeler S: Number and migratory activity of circulating endothelial progenitor cells inversely correlate with risk factors for coronary artery disease. Circ Res 89: E1-E7, 2001.

25. Asahara T, Masuda H, Takahashi T, Kalka C, Pastore C, Silver M, Kearne M, Magner M and Isner JM: Bone marrow origin of endothelial progenitor cells responsible for postnatal vasculogenesis in physiological and pathological neovascularization. Circ Res 85: 221-228, 1999.

26. Takahashi T, Kalka C, Masuda H, Chen D, Silver M, Kearney M, Magner M, Isner JM and Asahara T: Ischemia- and cytokineinduced mobilization of bone marrow-derived endothelial progenitor cells for neovascularization. Nat Med 5: 434-438, 1999.

27. He T, Smith LA, Harrington S, Nath KA, Caplice NM and Katusic ZS: Transplantation of circulating endothelial progenitor cells restores endothelial function of denuded rabbit carotid arteries. Stroke 35: 2378-2384, 2004.
28. Walter DH, Rittig K, Bahlmann FH, Kirchmair R, Silver M, Murayama T, Nishimura H, Losordo DW, Asahara T and Isner JM: Statin therapy accelerates reendothelialization: A novel effect involving mobilization and incorporation of bone marrow-derived endothelial progenitor cells. Circulation 105: 3017-3024, 2002.

29. Flora A, Garcia JJ, Thaller C and Zoghbi HY: The E-protein Tcf4 interacts with Math1 to regulate differentiation of a specific subset of neuronal progenitors. Proc Natl Acad Sci USA 104: 15382-15387, 2007

30. Wang Q, Lu PH, Shi ZF, Xu YJ, Xiang J, Wang YX, Deng LX, Xie P, Yin Y and Zhang B: Glucocorticoid receptor $\beta$ acts as a co-activator of T-cell factor 4 and enhances glioma cell proliferation. Mol Neurobiol 52: 1-13, 2014

31. Forrest MP, Hill MJ, Quantock AJ, Martin-Rendon E and Blake DJ: The emerging roles of TCF4 in disease and development. Trends Mol Med 20: 322-331, 2014.

32. Ciarapica R, Annibali D, Raimondi L, Savino M, Nasi S and Rota R: Targeting Id protein interactions by an engineered HLH domain induces human neuroblastoma cell differentiation. Oncogene 28: 1881-1891, 2009. 